\title{
Prevalence of Nocturnal Oxygen Desaturation in Subjects with HIV Infection
}

Linh Nguyen*, Juliana C Stradley, Mona White, Joanne Giordano, Sara Dingwall, John Suen and Gerald Pierone

Whole Family Health Center, Clinical Research Intern, USA

\begin{abstract}
Background: Obstructive sleep apnea (OSA) is a common disorder that is linked to daytime fatigue and increased risk of cardiovascular disease. The general population prevalence of OSA is estimated to be $2-9 \%$. The prevalence of OSA in people with HIV infection is unknown, although many patients with HIV report daytime fatigue. The diagnosis of OSA is typically established during an overnight study in a sleep laboratory. Sleep surveys designed to select have been previously validated for selecting patients for definitive study. Nocturnal oxygen desaturation index (ODI) measurement has also been used to screen for reliably predicting over $85 \%$ of moderate to severe cases when the ODI $>10$.
\end{abstract}

Methodology: Patients with HIV infection presenting for routine medical care were invited to participate in this prospective OSA screening trial. Informed consent was obtained; subjects completed the Four-Variable Screening Tool, the STOP-BANG questionnaire, Epworth Sleepiness Scale, and were provided a fingertip oximeter for overnight use at home. Overnight oxygen saturation data was analyzed and correlated to corresponding sleep surveys. All subjects with ODI >10 were referred for a formal sleep study.

Results: 156 subjects completed the study and 66 (42.3\%) demonstrated an ODI >10. Of the 66, 21 pursued formal sleep studies. 14 of the 21 tested positive for OSA. The Four-Variable Screening Tool had the highest positive predictive value of $59.5 \%$, and the STOP-BANG questionnaire had the highest negative predictive value of $76.9 \%$.

Conclusions: HIV-infected patients in our clinic demonstrated a high prevalence of nocturnal oxygen desaturation suggesting a high prevalence of OSA. Three commonly used screening tools did not reliably predict nocturnal desaturation in our population. The high prevalence of nocturnal oxygen desaturation and in HIV patients indicates the need for additional HIV-related OSA research.

Keywords: Desaturation; ODI; OSA; HIV; STOP-BANG; Epworth sleepiness scale; Four-variable screening Tool

\section{Introduction}

Nocturnal oxygen desaturation has been shown to be a reliable indicator of sleep disorders [1]. By using a fingertip oximeter to measure the oxygen saturation levels during an individual's nocturnal sleep cycle, episodes of significantly decreased blood oxygen levels can be quantified. The length and severity of these desaturation events can be used to predict whether or not an individual is at risk for a sleep disorder.

Obstructive sleep apnea (OSA) is a specific sleep disorder that is characterized by obstructive apneas and hypopneas caused by repetitive collapse of the upper airway during sleep. The severity of OSA is traditionally measured and defined by the apnea hypopnea index (AHI). This index quantifies the number of apneic and hypopneic events in an hour of sleep. There are various definitions of OSA. If OSA is defined having an AHI greater than 5 in conjunction with at least one symptom that responds to treatment, an estimated $2 \%-9 \%$ of the North American population may be affected [2,3].

One of the characteristics of OSA that negatively impacts quality of life is heightened daytime somnolence. Moreover, patients with OSA are also at risk for coronary heart disease, heart failure [4-6], stroke [7], hypoventilation syndrome [8], and chronic obstructive pulmonary disease [9].

Once diagnosed, measures such as the administration of continuous positive airway pressure (CPAP), bi-level positive airway pressure (BPAP), mandibular advancement devices (MAD), and/or supplemental oxygen may be utilized to reduce apneic episodes and improve quality of life.
Fatigue is an extremely common symptom in patients with HIV infection [10] and as many as $70 \%$ of patients with HIV infection suffer from sleep disturbances [11]. Despite this high statistic, there is scant literature about OSA in this patient population since the introduction of highly active antiretroviral therapy (HAART). Early studies linked HIV-induced adenotonsillar hypertrophy with the development of OSA [12]. Subsequent studies identified a significant prevalence of metabolic syndrome in patients with HIV infection [13]. The associated obesity and fat redistribution alter the morphology of the airways and thereby increase the risk of OSA [14].

An overnight sleep study is required to confirm a diagnosis of OSA. However, a variety of screening tests have been studied to identify patients most likely to require a formal overnight sleep studies. Examples include the Four-Variable Screening Tool, STOPBANG questionnaire, and the Epworth Sleepiness Scale $[1,15,16]$. In addition, overnight oximetry has been employed as a method to screen for OSA. By analyzing periods of oxygen desaturation during sleep and determining the average number of desaturation events per hour, quantified as the oxygen desaturation index (ODI), some studies suggest that it is possible to infer moderate to severe cases of OSA based

*Corresponding author: Linh Tan Nguyễn, Whole Family Health Center 981 37th Place, Vero Beach, FL, USA 32960, Tel: +1 (772) 538-9955; E-mail: Linh.Nguyen1024@gmail.com

Received February 18, 2016; Accepted May 14, 2016; Published May 21, 2016

Citation: Nguyen L, Stradley JC, White M, Giordano J, Dingwall S, et al. (2016) Prevalence of Nocturnal Oxygen Desaturation in Subjects with HIV Infection. $J$ AIDS Clin Res 7: 580. doi:10.4172/2155-6113.1000580

Copyright: $\odot 2016$ Nguyen L, et al. This is an open-access article distributed under the terms of the Creative Commons Attribution License, which permits unrestricted use, distribution, and reproduction in any medium, provided the original author and source are credited. 
on the data obtained from the oximetry. Previous studies have shown that ODI readings over 10 on overnight oximetry are predictive of moderate to severe sleep apnea [13].

Based on the evidence that supports nocturnal oxygen desaturation as a dependable indicator of OSA, we sought to utilize this method to determine the prevalence of nocturnal oxygen desaturation in subjects with HIV infection and subsequently investigate the efficacy of this index as a potential surrogate marker for OSA. We also administered the aforementioned three sleep surveys as alternative methods of screening for OSA and then correlated the results of these surveys with oxygen saturation data as an additional secondary objective.

\section{Materials and Methods}

This study was a pilot prospective nocturnal oximetry screening trial performed in two affiliated clinics specializing in HIV primary care. Patients presenting for routine medical care were offered the opportunity to participate in the study if they: (1) had HIV infection, and (2) were able to return the oximetry equipment by a reasonable date. Subjects were excluded if they already used supplemental oxygen, had a history of heart failure, stroke, or a resting oxygen saturation below $90 \%$. After obtaining informed consent, data was collected on each subject's demographics (gender, date of birth, race/ethnicity), vitals, (heart rate, blood pressure), weight ( $\mathrm{kg}$ ), height (inches), neck circumference (centimeters). From this data, each subject's body mass index $(\mathrm{BMI})\left(\mathrm{kg} / \mathrm{m}^{2}\right)$ was calculated.

Subjects were asked to complete three sleep screening tools, the Four-Variable Screening Tool, STOP-BANG questionnaire, and Epworth Sleepiness Scale. The STOP-BANG questionnaire (Table 1) consists of 8 simple yes/no questions regarding subjects' snoring habits, daytime somnolence, breathing during sleep, high blood pressure, BMI, age, neck circumference, and gender [1]. At least 3 answers of "yes" to the prompts are purported to be indicative of risk for OSA.

The Four-Variable Screening Tool (Table 2) produced a score based on each subject's gender, BMI, blood pressure, and self-reported frequency of snoring [15]. A score of at least 14 is purported to be indicative of sleep disordered breathing.

Subjects gauged their own likelihood of falling asleep in different scenarios set forth by the Epworth Sleepiness Scale (Table 3).

After completion of the screening surveys, subjects were then given oximeters to take home. The oximeters were worn during the nocturnal sleep cycle to measure and collect episodes of oxygen desaturation

\begin{tabular}{|l|l|l|l|}
\hline Snore & $\begin{array}{l}\text { Do you snore loudly (louder than talking or loud } \\
\text { enough to be heard through closed doors)? }\end{array}$ & Yes/No & Subscore \\
\hline Tired & $\begin{array}{l}\text { Do you often feel tired, fatigued, or sleepy } \\
\text { during daytime? }\end{array}$ & & \\
\hline $\begin{array}{l}\text { Observed } \\
\text { Apnea }\end{array}$ & $\begin{array}{l}\text { Has anyone observed you stop breathing } \\
\text { during your sleep? }\end{array}$ & & \\
\hline Pressure & $\begin{array}{l}\text { Do you have or are you being treated for high } \\
\text { blood pressure? }\end{array}$ & & \\
\hline BMI & Is your BMl more than $35 \mathrm{~kg} / \mathrm{m}^{2} ?$ & & \\
\hline Age & Are you over 50 years old? & & \\
\hline Neck & Is your neck circumference greater than $40 \mathrm{~cm} ?$ & & \\
\hline Gender & Are you male? & \\
\hline
\end{tabular}

Table 1: STOP-BANG Questionnaire. Values are assigned depending on the subject's answers, where a "yes" is assigned a value of 1 and a "no" is assigned a value of 0 . A score of at least 3 is indicative of risk for a sleeping disorder.

\begin{tabular}{|l|l|l|}
\hline & & Score \\
\hline Gender: & & \\
\hline BMI: & & \\
\hline Blood pressure: & & \\
\hline Frequency of snoring: & & \\
\hline
\end{tabular}

Table 2: Four-Variable Screening Tool. Values are assigned depending on the subject's answers. If the subject is male, a value of 4 is assigned; if the subject is female, a value of 0 is assigned. A BMI of less than 21.0 is assigned a value of 1 ; a BMI between 21.0-22.9 is assigned a value of 2; a BMI between $23.0-24.9$ is assigned a value of 3 ; a BMI between $25.0-26.9$ is assigned a value of 4 ; a BMI between $27.9-29.9$ is assigned a value of 5 ; a BMI of at least 30 is assigned a value of 6 . A systolic blood pressure (SBP) of less than 140 or a diastolic blood pressure (DBP) of less than 90 is assigned a value of 1 ; an SBP between $140-159$ or DBP between $90-99$ is assigned a value of 2; an SBP between $160-179$ or DBP between $100-109$ is assigned a value of 3; an SBP of at least 180 or a DBP of at least 110 is assigned a value of 4 . Nightly snoring is assigned a value of 4 ; all others are assigned a value of 0 . When the values total a score of at least 14 is indicative of sleep disordered breathing.

\begin{tabular}{|l|l|}
\hline Situation & $\begin{array}{c}\text { Chance of Dozing or } \\
\text { Sleeping }\end{array}$ \\
\hline Sitting and reading & \\
\hline Watching TV & \\
\hline Sitting inactive in public place (e.g., theater, meeting) & \\
\hline Passenger in a car for more than an hour without break & \\
\hline Lying down in the afternoon & \\
\hline Sitting and talking to someone & \\
\hline In a car while stopped for a few minutes in traffic & \\
\hline
\end{tabular}

Table 3: Epworth Sleepiness Scale. Subjects gauged their own likelihood of falling asleep in the assigned situations and assigned each situation a value ranging from 0 to 3 . If the subject would never fall asleep in the described scenario, a value of 0 is assigned. If there if a slight chance that the subject would fall asleep in the described scenario, a value of 1 is assigned. If there is a moderate chance that the subject would fall asleep in the described scenario, a value of 2 is assigned. If there is a high chance that subject would fall asleep in the described scenario, a value of 3 is assigned. When the values total a score of at least 10 , the subject may want to consider seeking medical attention.

during sleep. Subjects were asked to return the oximeters the next day, at which point they received fiscal compensation for their participation in the study. The oximetry data was downloaded into our database to be analyzed and correlated with corresponding sleep surveys.

In this study, an abnormal (or positive) oximetry study for nocturnal oxygen desaturation was an ODI greater than an average of 10 episodes per hour of monitored sleep time. A significant episode of oxygen desaturation in this study is defined as a decline in oxygen saturation of $4 \%$ or more from baseline. Baseline is defined as the mean level of oxygen saturation during the first 3 minutes of recording.

ODI was used to summarize the oxygen desaturation data. A subject with an ODI greater than 10 was considered at risk for OSA and was referred for a formal sleep study. Diagnoses of OSA were made according to the final results of a formal sleep study. The oxygen desaturation data was also correlated with corresponding sleep surveys.

\section{Results}

A total of 156 subjects (Table 4) were enrolled. 105 of the 156 (67.3\%) were male, and 51 of the $156(32.4 \%)$ were female. The average age of the subjects was 52.9 years.

66 (42.3\%) of the subjects exhibited an ODI greater than $10.74 .2 \%$ of those with abnormal oxygen desaturation indices were male, while $25.8 \%$ were female. $43.9 \%$ of these subjects were Black, $53.1 \%$ were White, and 3.0\% were either Hispanic/Latino or Native American or Other Pacific Islander. 


\begin{tabular}{|l|c|c|}
\hline Subject population, $\mathbf{N = 1 5 6}$ & & $\begin{array}{c}\text { Subjects with ODI } \\
\mathbf{2 1 0} \mathbf{n}=\mathbf{6 6}\end{array}$ \\
\hline Male & 105 & 49 \\
\hline Female & 51 & 17 \\
\hline Age (years), mean & $52.9(30-75)$ & $53.8(30-73)$ \\
\hline Age (years), median & 52 & 54.5 \\
\hline Ethnicity & & \\
\hline \multicolumn{1}{|c|}{ Black } & 55 & 29 \\
\hline \multicolumn{1}{|c|}{ Hispanic/Latino } & 6 & 1 \\
\hline \multicolumn{1}{|c|}{ Other } & 3 & 1 \\
\hline White & $28.1(15.5-66.1)$ & $28.9(18.5-66.1)$ \\
\hline BMI (kg/m²), mean & 27.2 & 27.5 \\
\hline BMI (kg/m²), median & $39.6(28.0-53.0)$ & $40.7(28.0-53.0)$ \\
\hline Neck circumference $(\mathrm{cm})$, mean & 39.4 & 40.0 \\
\hline Neck circumference $(\mathrm{cm})$, median & $12.6(0-59.3)$ & $23.6(10.5-59.3)$ \\
\hline ODI, mean & 8.45 & 20.6 \\
\hline ODI, median & & 35 \\
\hline
\end{tabular}

Table 4: Demographics of total subject population $(N=156)$ juxtaposed with demographics and oxygen desaturation data for subjects whose ODI >10 $(n=66)$.

\begin{tabular}{|l|c|c|c|c|}
\hline & Sensitivity & Specificity & $\begin{array}{c}\text { Positive } \\
\text { Predictive Value }\end{array}$ & $\begin{array}{c}\text { Negative } \\
\text { Predictive Value }\end{array}$ \\
\hline STOP-BANG & $86.4 \%$ & $33.3 \%$ & $48.7 \%$ & $76.9 \%$ \\
\hline 4-Variable & $33.3 \%$ & $83.3 \%$ & $59.5 \%$ & $63.0 \%$ \\
\hline Epworth & $50.8 \%$ & $58.2 \%$ & $46.5 \%$ & $62.5 \%$ \\
\hline
\end{tabular}

Table 5: Statistical analysis of the STOP-BANG questionnaire, Four-Variable Screening Tool, and Epworth Sleepiness Scale shows that none were useful in predicting oxygen desaturation trends.

The specificity and sensitivity of the sleep surveys were calculated in order to assess their efficacy in predicting ODI. The STOP-BANG questionnaire showed high sensitivity when correlated with the oxygen desaturation data, while the Four-Variable Screening Tool showed high specificity. The Epworth Sleepiness Scale showed poor sensitivity and specificity. Table 5 summarizes the statistical analysis of the sleep survey.

Twenty-one of the 66 subjects with significant oxygen desaturation completed formal sleep studies. Fourteen of these 21 subjects (66\%) tested positive for OSA. Eight of these subjects had mild OSA, 4 had moderate OSA, and 2 had severe OSA. One of the subjects with severe OSA and another with moderate OSA reported notable improvement with the utilization of CPAP.

Four additional subjects were recommended for formal sleep studies due to significant oxygen desaturation and clinical findings which suggested a high likelihood of a possible sleep disorder despite exhibiting an ODI less than 10. One of these patients tested positive for OSA and received CPAP with significant improvement. Another received nocturnal supplementary oxygen.

\section{Discussion}

Nearly half of the subjects with HIV infection in this study exhibited significant oxygen desaturations during their nocturnal sleep cycle. This substantial proportion of subjects suggests that the prevalence of disregulated sleeping may be much higher in subjects with HIV infection as compared with un-infected individuals. When compared to a similar study with 19 less subjects, our preliminary findings reveal a similar prevalence of OSA in patients with HIV. However, it must be noted that this pilot study faced limitations that, if overcome, could likely reveal a higher prevalence of OSA. The number of patients who completed follow up and obtained OSA diagnoses supports the viability of ODI as a screening tool. A comparison to previous studies which explored BMI and neck circumference as anatomical markers for OSA revealed that subjects with significant desaturation indices in this study did not present with as notable of physical features [17]. This incidental finding suggests that physical presentation is not adequate criteria alone and supports the need for a screening method simpler than a formal sleep study.

The scores from STOP-BANG questionnaire, Four-Variable Screening Tool, and Epworth Sleepiness Scale were shown to be incongruent with the corresponding oximetry data. A statistical analysis showed that all three surveys proved to be unhelpful, as none could accurate predict which subjects were more likely to experience significant desaturation events. While the STOP-BANG questionnaire showed a high degree of sensitivity, the results were accompanied by a low rate of specificity. Inversely, the Four-Variable Screening Tool exhibited high specificity in conjunction with low sensitivity. The Epworth Sleepiness Scale performed poorly in regards to both sensitivity and specificity.

The positive predictive value (PPV) of the 4 -Variable Screen Tool proved to be only slightly better than chance while the PPV for the STOP-BANG questionnaire and Epworth Sleepiness Scale displayed even worse predictive ability. All three screening surveys showed unremarkable negative predictive ability, as exemplified by their respective negative predictive values (NPV). In all cases, no sleep survey proved to be reliably accurate or useful in predicting oxygen desaturation trends in our subject population and are therefore unreliable tools to be used for screening of sleep disorders in our subject population, even in cases of extreme oxygen desaturation. The failure of these questionnaires suggest that nocturnal oximetry might serve as a better screening tool for individuals with HIV at risk for sleep disorders.

All 66 subjects with significant oxygen desaturations were encouraged to follow up with a formal overnight sleep study, but only 21 completed a sleep study, thereby limiting the results and conclusions of our pilot study. The reason that some subjects declined further study was because they were asymptomatic and unmotivated. Other subjects choose not to follow through because of financial barriers and co-pay costs of the formal sleep study.

Fourteen of the 21 subjects who followed up with formal sleep studies were found to have obstructive sleep apnea. But only 8 of the 21 subjects were found to have moderate to severe obstructive sleep apnea. This relatively low yield of subjects identified as having moderate to severe obstructive sleep apnea is in contrast with previous studies in which the majority of patients with ODI greater than 10 had moderate to severe obstructive sleep apnea.

Several subjects obtained a CPAP device and reported improvements in their nocturnal sleep cycle and improvements in daytime somnolence. Some subjects who were not able to tolerate CPAP, but were treated with supplemental oxygen. Also, several subjects exhibiting significant oxygen desaturation were diagnosed with COPD, which resulted in therapeutic intervention with supplemental oxygen.

Although the oximetry data and sleep disordered breathing diagnoses obtained by subjects who underwent formal sleep studies show potential for oximetry as a reliable screening tool, additional formal sleep studies are still required to validate nocturnal oximetry 
as an accurate indicator of sleep disorders in patients with HIV infection.

\section{Conclusions}

Our study showed a high prevalence of nocturnal oxygen desaturation in subjects with HIV infection. This finding suggests that the prevalence of obstructive sleep apnea in patients with HIV infection may be high as well.

We also found that three previously validated screening tools for OSA were not predictive of nocturnal oxygen desaturation in our study population.

Given the nature of our pilot study we did not have enough data from the subset of patients who completed formal sleep studies to determine whether overnight oximetry is a reliable predictor of moderate to severe obstructive sleep apnea in patients with HIV infection, as our study was limited secondary to subject follow-up. However, we believe that the observed high prevalence of oxygen desaturation warrants further investigation to validate oxygen desaturation as an alternative marker for OSA. Due to the substantial time commitment and fiscal burden associated with a polysomnography test, we recommend the provision of subsidies in order to encourage greater follow up on future studies. Furthermore, we would suggest correlating subjects' ODI with HAART regimen and lab values such as viral load and CD4 count.

The small study population of our pilot study limited our capacity to perform a formal statistical analysis. Further studies would be strengthened by including a comparison of the test group to a control group without HIV infection. Additional study will be necessary to follow up on our findings of the high prevalence of overnight oxygen desaturation associated with HIV infection. The inclusion of a control group in conjunction with further follow up would expand the subject population to allow for significant statistical analysis.

\section{References}

1. Takegami M, Hayashino Y, Chin K, Sokejima S, Kadotani H, et al. (2009) Simple four-variable screening tool for identification of patients with sleep-disordered breathing. Sleep 32: 939-948.

2. Young T, Palta M, Dempsey J, Peppard PE, Nieto FJ, et al. (2009) Burden of sleep apnea: rationale, design, and major findings of the Wisconsin Sleep Cohort study. WMJ 108: 246-249.

3. Dempsey JA, Veasey SC, Morgan BJ, O'Donnell CP (2010) Pathophysiology of sleep apnea. Physiol Rev 90: 47-112.
4. Somers VK, White DP, Amin R, Abraham WT, Costa F, et al. (2008) Sleep apnea and cardiovascular disease: an American Heart Association/American College Of Cardiology Foundation Scientific Statement from the American Heart Association Council for High Blood Pressure Research Professional Education Committee, Council on Clinical Cardiology, Stroke Council, and Council On Cardiovascular Nursing. In collaboration with the National Heart, Lung, and Blood Institute National Center on Sleep Disorders Research (National Institutes of Health). Circulation 118: 1080-1111.

5. Gottlieb DJ, Yenokyan G, Newman AB, O'Connor GT, Punjabi NM, et al. (2010) Prospective study of obstructive sleep apnea and incident coronary heart disease and heart failure: the sleep heart health study. Circulation 122: 352360.

6. Loke YK, Brown JW, Kwok CS, Niruban A, Myint PK (2012) Association of obstructive sleep apnea with risk of serious cardiovascular events: a systematic review and meta-analysis. Circ Cardiovasc Qual Outcomes 5: 720-728.

7. Yaggi HK, Concato J, Kernan WN, Lichtman JH, Brass LM, et al. (2005) Obstructive sleep apnea as a risk factor for stroke and death. N Engl J Med 353: 2034-2041.

8. Mokhlesi B, Kryger MH, Grunstein RR (2008) Assessment and management of patients with obesity hypoventilation syndrome. Proc Am Thorac Soc 5: 218225.

9. Martinez $\mathrm{CH}$, Han MK (2012) Contribution of the environment and comorbidities to chronic obstructive pulmonary disease phenotypes. Med Clin North Am 96: 713-727.

10. Barroso J, Voss J (2013) Fatigue in HIV and AIDS: An Analysis of Evidence. Journal Of The Association Of Nurses In AIDS Care 24: S5-S14.

11. Diana MT (2013) Sleep Disturbances in Persons Living with HIV. J Assoc Nurses AIDS Care 24: S72-S85.

12. Epstein LJ, Strollo PJ Jr, Donegan RB, Delmar J, Hendrix C, et al. (1995) Obstructive sleep apnea in patients with human immunodeficiency virus (HIV) disease. Sleep 18: 368-376.

13. Cooper BG, Veale D, Griffiths CJ, Gibson GJ (1991) Value of nocturnal oxygen saturation as a screening test for sleep apnoea. Thorax 46: 586-588.

14. TSH, MEJL (2013) Obesity and metabolic complications: Metabolic syndrome. Medicine 39: 24-31.

15. Chung F, Subramanyam R, Liao P, Sasaki E, Shapiro C, et al. (2012) High STOP-Bang score indicates a high probability of obstructive sleep apnoea. $\mathrm{Br}$ J Anaesth 108: 768-775.

16. Haddad R, AlSureehein S, Alsamen G, Alzboon M, Al Hazeem A (2015) Evaluation of Epworth Sleepiness Scale as a screening method for Obstructive Sleep Apnea Syndrome. Middle East Journal of Internal Medicine 8: 3-6.

17. Lo Re V 3rd, Schutte-Rodin S, Kostman JR (2006) Obstructive sleep apnoea among HIV patients. Int J STD AIDS 17: 614-620. 